\title{
Supporting Information: Anharmonic Correction to Adsorption Free Energy from DFT-based MD using Thermodynamic Integration
}

\author{
Jonas Amsler, ${ }^{*, \dagger}$ Philipp N. Plessow, ${ }^{\dagger}$ Felix Studt, ${ }^{\dagger, \ddagger}$ and Tomáš Bučko, ${ }^{*, \S}$ \\ $\dagger$ Institute of Catalysis Research and Technology, Karlsruhe Institute of Technology, \\ Hermann-von-Helmholtz-Platz 1, 76344 Eggenstein-Leopoldshafen, Germany \\ $\ddagger$ Institute for Chemical Technology and Polymer Chemistry, Karlsruhe Institute of \\ Technology, Kaiserstr. 12, 76131 Karlsruhe, Germany \\ IDepartment of Physical and Theoretical Chemistry, Faculty of Natural Sciences, \\ Comenius University in Bratislava, Ilkovičova 6, SK-84215 Bratislava, Slovakia \\ §Institute of Inorganic Chemistry, Slovak Academy of Sciences, Dúbravská cesta 9, \\ SK-84236 Bratislava, Slovakia
}

E-mail: jonas.amsler@kit.edu; bucko19@uniba.sk 


\section{Contents}

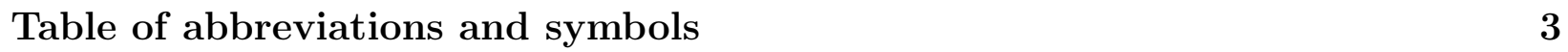

$\begin{array}{lll}\text { S1 Coordinate transformation solving the rotational problem (example) } & 7\end{array}$

$\begin{array}{ll}\text { S2 Classical TI vs. quantum solutions } & 10\end{array}$

S2.1 Comparison of classical free energies of model systems with quantum mechanical solutions ......................... . . . 10

S2.1.1 HF molecule. . . . . . . . . . . . . . . . . . 10

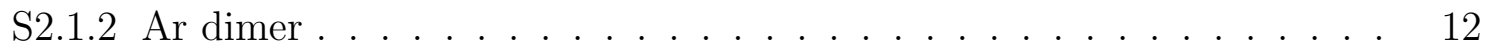

S2.1.3 Ethane molecule . . . . . . . . . . . . . . . . . . . . 12

S2.2 Classical vs. quantum harmonic approximation for adsorption problems . . . 15

$\begin{array}{ll}\text { S3 Computational details } & 17\end{array}$

S3.1 Numerical integration . . . . . . . . . . . . . . . . . 17

S3.2 Statistical error estimation . . . . . . . . . . . . . . . 18

S3.3 Structural model for the adsorption studies . . . . . . . . . . . . . . . . 18

$\begin{array}{ll}\text { S4 Regression of experimental adsorption free energies } & 19\end{array}$

$\begin{array}{ll}\text { S5 Practical information } & 20\end{array}$

S5.1 How to perform a TI MD in VASP - a brief workflow . . . . . . . . . . 20

$\begin{array}{lr}\text { References } & 21\end{array}$ 


\section{Table of abbreviations and symbols}

Table S1: Meaning of abbreviations and symbols.

\begin{tabular}{|c|c|}
\hline Symbol & Meaning \\
\hline 0 & the reference system of the $\lambda$-path integration \\
\hline 1 & the target system of the $\lambda$-path integration \\
\hline$a_{i}$ & $i$ th lattice vector \\
\hline$A_{\mathrm{el}}$ & electronic free energy (DFT) \\
\hline$A_{0, \boldsymbol{x}}$ & Helmholtz free energy of system harmonic in $\boldsymbol{x}$ \\
\hline$A_{0, \boldsymbol{q}}$ & Helmholtz free energy of system harmonic in $\boldsymbol{q}$ \\
\hline$A_{1}$ & Helmholtz free energy of fully interacting system (DFT) \\
\hline$\Delta A_{0 \rightarrow 1}$ & total anharmonic Helmholtz free energy correction $\left(\Delta A_{0 \rightarrow 1} \equiv \Delta A_{0, \boldsymbol{x} \rightarrow 1}\right)$ \\
\hline$\Delta A_{0, \boldsymbol{x} \rightarrow 0, \boldsymbol{q}}$ & anharmonic correction via TI of harmonic coordinate transformation \\
\hline$\Delta A_{0, \boldsymbol{q} \rightarrow 1}$ & anharmonic correction via TI from harmonic to DFT potential \\
\hline A & system with adsorbate in gas-phase (no substrate) \\
\hline $\mathrm{A} @ \mathrm{~S}$ & system with adsorbate and substrate \\
\hline$\underline{A}$ & Moore-Penrose pseudo-inverse of $\underline{\boldsymbol{B}}$ \\
\hline$\underline{B}$ & Wilson B-matrix \\
\hline corr. & correction \\
\hline$c_{n}$ & expansion coefficients \\
\hline$C$ & force constant \\
\hline$C N$ & coordination number \\
\hline $\mathrm{CV}$ & Collective Variable \\
\hline$d_{i}$ & distance between rotation axis and atom $i$ \\
\hline $\mathrm{DFT}$ & Density Functional Theory \\
\hline $\mathrm{D}$ & dimensional \\
\hline$f^{x}$ & forces in Cartesian coordinates \\
\hline
\end{tabular}




\begin{tabular}{|c|c|}
\hline Symbol & Meaning \\
\hline$f^{q}$ & forces in internal coordinates \\
\hline$g_{j}$ & multiplicity of the electronic level $j$ \\
\hline$G_{0, A}$ & $\begin{array}{l}\text { Gibbs free energy of adsorbate in the ideal gas free translational state, rigid } \\
\text { rotor and harmonic oscillator approximation }\end{array}$ \\
\hline$\Delta_{\mathrm{ads}} G$ & adsorption Gibbs free energy \\
\hline$\hbar$ & Planck constant divided by $2 \pi$ \\
\hline$\underline{H}^{x}$ & Hessian matrix in Cartesian coordinates \\
\hline$\underline{H}^{q}$ & Hessian matrix in internal coordinates \\
\hline $\mathcal{H}_{0, \boldsymbol{x}}$ & Hamiltonian of system harmonic in $\boldsymbol{x}$ \\
\hline $\mathcal{H}_{0, \boldsymbol{q}}$ & Hamiltonian of system harmonic in $\boldsymbol{q}$ \\
\hline $\mathcal{H}_{1}$ & Hamiltonian of fully interacting system (DFT: PBE-D2) \\
\hline $\mathcal{H}_{\lambda}$ & $\begin{array}{l}\text { Hamiltonian for a virtual system defined by the value of the coupling pa- } \\
\text { rameter } \lambda\end{array}$ \\
\hline HA & Harmonic Approximation \\
\hline$I$ & moment of inertia \\
\hline$k_{\mathrm{B}}$ & Boltzmann constant \\
\hline$L$ & $\begin{array}{l}\text { triplet of integers representing translation along the three lattice vectors } \\
\text { defining the unit cell }\end{array}$ \\
\hline$m_{i}$ & mass of atom $i$ \\
\hline MD & Molecular Dynamics (simulation) \\
\hline $\mathrm{ML}$ & machine learning \\
\hline$N_{\text {sub }}$ & number of substrate atoms considered in $\mathrm{CN}$ \\
\hline$N_{\text {vib }}$ & number of vibrational degrees of freedom \\
\hline NVT & canonical ensemble with constant number of particles, volume and $\mathrm{T}$ \\
\hline$p V$ & product of pressure and volume \\
\hline$p$ & momenta \\
\hline
\end{tabular}




\begin{tabular}{|c|c|}
\hline Symbol & Meaning \\
\hline PAW & Projector Augmented Wave (method) \\
\hline$q$ & internal coordinates \\
\hline$q_{0}$ & internal coordinates corresponding to reference geometry \\
\hline$q_{\mathrm{hr}}$ & partition function of $1 \mathrm{D}$ hindered rotor \\
\hline$q_{\text {rot }}$ & molecular rotational partition function \\
\hline$q_{\text {vib }}$ & molecular vibrational partition function \\
\hline QHA & Quasi-Harmonic Approximation \\
\hline$r_{i j, L}$ & distance between atoms $i$ and $j$ \\
\hline$R_{i j}$ & reference distance between atoms $i$ and $j$ \\
\hline$R$ & distance \\
\hline $\mathrm{RDF}$ & radial distribution function \\
\hline $\mathrm{S}$ & system with clean substrate (no adsorbate) \\
\hline SI & Supporting Information \\
\hline SOAP & smooth overlap of atomic positions \\
\hline$T$ & temperature \\
\hline TI & Thermodynamic Integration (here: $\lambda$-path integration) \\
\hline$V$ & potential energy \\
\hline$V_{0, \boldsymbol{x}}$ & potential energy harmonic in $\boldsymbol{x}$ \\
\hline$V_{0, \boldsymbol{q}}$ & potential energy harmonic in $\boldsymbol{q}$ \\
\hline$V_{1}$ & potential energy of a fully interacting system (DFT: PBE-D2) \\
\hline VAF & velocity autocorrelation function \\
\hline VASP & Vienna Ab Initio Simulation Package \\
\hline VDOS & vibrational density of states \\
\hline$W$ & potential energy barrier for the internal rotation \\
\hline$x_{1}, x_{2}$ & components of Cartesian coordinates \\
\hline $\boldsymbol{x}$ & Cartesian coordinates \\
\hline
\end{tabular}




\begin{tabular}{|c|c|}
\hline Symbol & Meaning \\
\hline $\boldsymbol{x}_{0}$ & Cartesian coordinates corresponding to reference geometry \\
\hline$\tilde{\boldsymbol{x}}_{0}$ & Cartesian coordinates corresponding to re-oriented reference geometry \\
\hline$\alpha$ & angle \\
\hline$\alpha_{i}$ & coefficient from the Simpson integration scheme \\
\hline$\beta$ & $\frac{1}{k_{B} T}$ \\
\hline$\beta_{i}$ & coefficient from the Simpson integration scheme \\
\hline$\varepsilon_{j}$ & eigenenergy of the state $j$ \\
\hline$\eta_{i}$ & coefficient from the Simpson integration scheme \\
\hline$\lambda$ & coupling parameter, $\lambda \in[0,1]$ \\
\hline$\mu$ & reduced mass \\
\hline$\xi$ & reaction coordinate \\
\hline$\sigma$ & symmetry number \\
\hline$\sigma_{r}$ & symmetry number of internal rotation \\
\hline$\tau$ & torsion angle (dihedral) \\
\hline$\tau_{0}$ & torsion angle (dihedral) corresponding to reference geometry \\
\hline$\chi_{\mathrm{e}}$ & first anharmonicity constant \\
\hline$\Psi$ & wave function \\
\hline$\omega$ & harmonic frequency \\
\hline
\end{tabular}




\section{S1 Coordinate transformation solving the rotational problem (example)}

The following illustration is intended to demonstrate the usefulness of a coordinate transformation for solving the rotational problem. Consider a harmonic spring with force constant $C$, equilibration length $R_{0}$ and instantaneous length $R$ as sketched in Figure S1. The spring
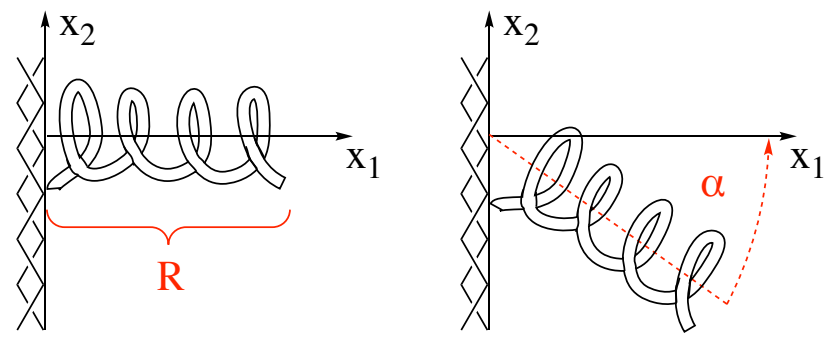

Figure S1: Left: Harmonic spring attached to a rigid wall. Right: Harmonic spring rotated by an angle $\alpha$ (note that here $\alpha<0)$.

existing in $2 \mathrm{D}$ is attached to a rigid wall while the other end is free. The position vector $\boldsymbol{x}$ and the equilibrium position vector $\boldsymbol{x}_{0}$ are defined as

$$
\boldsymbol{x}=\left(\begin{array}{c}
x_{1} \\
x_{2}
\end{array}\right)=\left(\begin{array}{c}
R \\
0
\end{array}\right) \quad \text { and } \quad \boldsymbol{x}_{0}=\left(\begin{array}{c}
x_{1,0} \\
x_{2,0}
\end{array}\right)=\left(\begin{array}{c}
R_{0} \\
0
\end{array}\right)
$$

The force acting on the free end point of the spring in Cartesian coordinates can be obtained from the Cartesian Hessian matrix $\underline{\boldsymbol{H}}^{\boldsymbol{x}}$ and the displacement from the equilibration position:

$$
\begin{aligned}
\boldsymbol{f}^{\boldsymbol{x}} & =-\underline{\underline{\boldsymbol{H}}}^{\boldsymbol{x}}\left(\boldsymbol{x}-\boldsymbol{x}_{0}\right)=-\left(\begin{array}{ll}
C & 0 \\
0 & 0
\end{array}\right)\left(\begin{array}{c}
R-R_{0} \\
0
\end{array}\right)=-\left(\begin{array}{cc}
C\left(R-R_{0}\right) \\
0
\end{array}\right) \\
\text { with } \quad \underline{\boldsymbol{H}}^{\boldsymbol{x}} & =\left(\begin{array}{ll}
C & 0 \\
0 & 0
\end{array}\right) .
\end{aligned}
$$

Let us now consider the same spring rotated by an angle $\alpha$, without changing $R$. The 
new position $\boldsymbol{x}^{\prime}$ is obtained by application of the rotation matrix:

$$
\boldsymbol{x}^{\prime}=\left(\begin{array}{cc}
\cos \alpha & -\sin \alpha \\
\sin \alpha & \cos \alpha
\end{array}\right) \boldsymbol{x}=\left(\begin{array}{cc}
\cos \alpha & -\sin \alpha \\
\sin \alpha & \cos \alpha
\end{array}\right)\left(\begin{array}{l}
R \\
0
\end{array}\right)=\left(\begin{array}{c}
R \cos \alpha \\
R \sin \alpha
\end{array}\right)
$$

Since the force is a covector, it must transform just like $\boldsymbol{x}$ upon rotation, i.e.,

$$
\begin{aligned}
\boldsymbol{f}^{\boldsymbol{x} \prime} & =\left(\begin{array}{cc}
\cos \alpha & -\sin \alpha \\
\sin \alpha & \cos \alpha
\end{array}\right) \boldsymbol{f}^{\boldsymbol{x}}=-\left(\begin{array}{cc}
\cos \alpha & -\sin \alpha \\
\sin \alpha & \cos \alpha
\end{array}\right)\left(\begin{array}{c}
C\left(R-R_{0}\right) \\
0
\end{array}\right) \\
& =-C\left(R-R_{0}\right)\left(\begin{array}{c}
\cos \alpha \\
\sin \alpha
\end{array}\right) .
\end{aligned}
$$

Note that $\left|\boldsymbol{f}^{\boldsymbol{x}}\right|=\left|\boldsymbol{f}^{\boldsymbol{x}}\right|$ as it should be since the rotation cannot change the magnitude of the force. However, a naive usage of the Hessian matrix leads to incorrect forces:

$$
\boldsymbol{f}_{\text {naive }}^{\boldsymbol{x}^{\prime}}=-\underline{\boldsymbol{H}}^{\boldsymbol{x}}\left(\boldsymbol{x}^{\prime}-\boldsymbol{x}_{0}\right)=-\left(\begin{array}{cc}
C & 0 \\
0 & 0
\end{array}\right)\left(\begin{array}{c}
R \cos \alpha-R_{0} \\
R \sin \alpha
\end{array}\right)=-\left(\begin{array}{c}
C\left(R \cos \alpha-R_{0}\right) \\
0
\end{array}\right)
$$

This is obviously wrong since $\left|\boldsymbol{f}_{\text {naive }}^{x^{\prime}}\right| \neq\left|\boldsymbol{f}^{x}\right|$ for $\alpha \neq 0$.

Interestingly, the problem is tackled easily by the coordinate transformation $\boldsymbol{x} \rightarrow \boldsymbol{q}$, i.e., $\left\{x_{1}, x_{2}\right\} \rightarrow\{R\}$ through which the forces in Cartesian coordinates $\boldsymbol{x}$ can be obtained from the forces in internal coordinates $\boldsymbol{q}$. The coordinate transformation of the Hessian matrix $\left(\underline{\boldsymbol{H}}^{\boldsymbol{x}} \rightarrow \underline{\boldsymbol{H}}^{\boldsymbol{q}}\right)$ is achieved via the transformation relation ${ }^{1-5}$

$$
\underline{H}^{\boldsymbol{q}}=\underline{\boldsymbol{A}}_{\boldsymbol{x}_{0}}^{T} \underline{\boldsymbol{H}}^{\boldsymbol{x}} \underline{\boldsymbol{A}}_{\boldsymbol{x}_{0}}
$$

where $\underline{\boldsymbol{A}}_{\boldsymbol{x}_{0}}$ is the Moore-Penrose pseudo-inverse of the Wilson B-Matrix $\underline{\boldsymbol{B}}_{\boldsymbol{x}_{0}}$ for the relaxed 
structure:

$$
\begin{aligned}
& \underline{\boldsymbol{B}}_{\boldsymbol{x}_{0}}=\left(\left.\left.\frac{\partial R}{\partial x_{1}}\right|_{\boldsymbol{x}_{0}} \frac{\partial R}{\partial x_{2}}\right|_{\boldsymbol{x}_{0}}\right)=\left(\begin{array}{ll}
1 & 0
\end{array}\right) \\
& \underline{\boldsymbol{A}}_{\boldsymbol{x}_{0}}=\left(\underline{\boldsymbol{B}}_{\boldsymbol{x}_{0}}^{T} \underline{\boldsymbol{B}}_{\boldsymbol{x}_{0}}\right)^{-1} \underline{\boldsymbol{B}}_{\boldsymbol{x}_{0}}^{T}=\left(\left(\begin{array}{l}
1 \\
0
\end{array}\right)\left(\begin{array}{ll}
1 & 0
\end{array}\right)\right)^{-1}\left(\begin{array}{l}
1 \\
0
\end{array}\right)=\left(\begin{array}{ll}
1 & 0 \\
0 & 0
\end{array}\right)^{-1}\left(\begin{array}{l}
1 \\
0
\end{array}\right)=\left(\begin{array}{l}
1 \\
0
\end{array}\right) .
\end{aligned}
$$

Combining eq. (S7) with eq. (S8) we obtain

$$
\underline{\boldsymbol{H}}^{\boldsymbol{q}}=\left(\begin{array}{ll}
1 & 0
\end{array}\right)\left(\begin{array}{ll}
C & 0 \\
0 & 0
\end{array}\right)\left(\begin{array}{l}
1 \\
0
\end{array}\right)=\left(\begin{array}{ll}
C & 0
\end{array}\right)\left(\begin{array}{l}
1 \\
0
\end{array}\right)=C .
$$

The force expressed in internal coordinates

$$
\boldsymbol{f}^{\boldsymbol{q}}=-\underline{\boldsymbol{H}}^{\boldsymbol{q}}\left(\boldsymbol{q}-\boldsymbol{q}_{0}\right)=-C\left(R-R_{0}\right)
$$

can subsequently be transformed back to Cartesian coordinates as follows: ${ }^{1-5}$

$$
\boldsymbol{f}^{\boldsymbol{x}^{\prime}}=\underline{\boldsymbol{B}}_{\boldsymbol{x}}^{T} \boldsymbol{f}^{\boldsymbol{q}}=-\left(\begin{array}{c}
\left.\frac{\partial R}{\partial x_{1}}\right|_{\boldsymbol{x}^{\prime}} \\
\left.\frac{\partial R}{\partial x_{2}}\right|_{\boldsymbol{x}^{\prime}}
\end{array}\right) C\left(R-R_{0}\right)=-C\left(R-R_{0}\right)\left(\begin{array}{c}
\cos \alpha \\
\sin \alpha
\end{array}\right)
$$

where we made use of the relations $\left.\frac{\partial R}{\partial x_{1}}\right|_{\boldsymbol{x}^{\prime}}=\frac{x_{1}^{\prime}}{R}=\cos \alpha$ and $\left.\frac{\partial R}{\partial x_{2}}\right|_{\boldsymbol{x}^{\prime}}=\frac{x_{2}^{\prime}}{R}=\sin \alpha$. By comparison with eq. (S5) we see that the force expression obtained by eq. (S12) yields the correct result. 


\section{S2 Classical TI vs. quantum solutions}

\section{S2.1 Comparison of classical free energies of model systems with quantum mechanical solutions}

The main purpose of the model systems presented in Section 3.1 of our article is to demonstrate the validity of our TI approach. Besides TI, the classical anharmonic correction for these systems can be determined in an alternative and independent way presented in the main text as a semi-analytic solution. The validation of our approach then consists of a direct comparison of its results against the semi-analytical reference. Albeit the following comparison with the exact quantum solutions is irrelevant in this respect, it nevertheless is interesting from a conceptual point of view.

\section{S2.1.1 HF molecule}

In order to access the quantum mechanical solution for the HF molecule, we adapted the commonly used approximation that individual rotational and vibrational degrees of freedom can be treated as independent motions. With the rotational and vibrational partition functions $\left(q_{\text {rot }}\right.$ and $\left.q_{\mathrm{vib}}\right)$ the free energy difference between the harmonic reference $(0)$ and the fully interacting (1) system can be computed via

$$
\Delta A_{0, \boldsymbol{x} \rightarrow 1}=\Delta A_{0, \boldsymbol{q} \rightarrow 1}=-k_{\mathrm{B}} T \ln \frac{q_{\mathrm{rot}, 1} q_{\mathrm{vib}, 1}}{q_{\mathrm{rot}, 0} q_{\mathrm{vib}, 0}}
$$

Note that the systems 0 and 1 do not differ in translational degrees of freedom. Hence, the corresponding partition functions cancel in the expression in eq. (S13). The quantum mechanical expressions for the vibrational partition functions of the harmonic oscillator 
$\left(q_{\mathrm{vib}, 0}\right)$ and the fully interacting system $\left(q_{\mathrm{vib}, 1}\right)$ are obtained via ${ }^{6}$

$$
q_{\mathrm{vib}, 0}=\frac{\exp \left(-\frac{\hbar \omega}{2 k_{\mathrm{B}} T}\right)}{1-\exp \left(-\frac{\hbar \omega}{k_{\mathrm{B}} T}\right)} \quad \text { and } \quad q_{\mathrm{vib}, 1}=\sum_{j} g_{j} \exp \left(-\beta \varepsilon_{j}\right)
$$

with the harmonic frequency $\omega=72.8 \mathrm{THz}$, the degeneracy $g_{j}$ of quantum state $j$, and $\varepsilon_{j}$ being the energy obtained by numerically solving the 1D Schrödinger eq. (S15) by the Fourier grid Hamiltonian method. ${ }^{7}$

$$
-\frac{\hbar^{2}}{2 \mu} \frac{d^{2}}{d R^{2}} \Psi+V_{1}(R) \Psi=\varepsilon \Psi
$$

A reduced mass of $\mu=4.30 \cdot 10^{-27} \mathrm{~kg}$ is obtained for the HF molecule using the mass of tritium instead of protium, as described in Section 2.2. The full potential (Figure 2) was expressed in the form of the inverse power expansion $V_{1}=\sum_{n=0}^{6} c_{n} R^{-n}$ whereby the expansion coefficients $c_{n}\left(\right.$ in $\left.\mathrm{eV} \AA^{\mathrm{n}+1}:-17.63,177.91,-496.64,660.52,-481.49,187.48,-30.06\right)$ have been obtained by fitting the potential energies evaluated on a regular 1D grid with 129 grid points. With $T=2000 \mathrm{~K}$ we obtain $\frac{q_{\mathrm{vib}, 1}}{q_{\mathrm{vib}, 0}}=1.0157$. For the rotational degrees of freedom, we adapted the commonly used rigid rotor approximation for our system with two equivalent rotational axes: ${ }^{6}$

$$
q_{\mathrm{rot}, \lambda}=\frac{2 \pi k_{\mathrm{B}} T I_{\lambda}}{\sigma \hbar^{2}}
$$

with the symmetry number $\sigma=1$ and the moment of inertia, $I_{\lambda}=\mu R_{\mathrm{eq}, \lambda}^{2}$, where $R_{\mathrm{eq}, \lambda}$ is the equilibrium distance between the $\mathrm{H}$ and $\mathrm{F}$ atoms evaluated for the harmonic $(\lambda=0)$ and the anharmonic $(\lambda=1)$ system. Since the expressions for $q_{\mathrm{rot}, 0}$ and $q_{\mathrm{rot}, 1}$ differ only in $I$, the fraction $\frac{q_{\mathrm{rot}, 1}}{q_{\mathrm{rot}, 0}}$ reduces to $\frac{R_{\mathrm{eq}, 1}^{2}}{R_{\mathrm{eq}, 0}^{2}}$. Due to the symmetry of the problem we express the partition function in spherical coordinates:

$$
R_{\mathrm{eq}, \lambda}=\frac{\int d R R^{3} \exp \left(-\beta V_{\lambda}(R)\right)}{\int d R R^{2} \exp \left(-\beta V_{\lambda}(R)\right)}
$$


and substitute $R_{\text {eq }, 0}$ and $R_{\text {eq }, 1}$ by the average inter-atomic distances $0.9447 \AA$ and $0.9581 \AA$, respectively, to find $\frac{q_{\mathrm{rot}, 1}}{q_{\mathrm{rot}, 0}}=1.0286$. Combining all terms we obtain $\Delta A_{0, \boldsymbol{x} \rightarrow 1}=\Delta A_{0, \boldsymbol{q} \rightarrow 1}=$ $-0.0075 \mathrm{eV}$ which is in excellent agreement with the reference value of $-7.6 \mathrm{meV}$ reported in our article. Finally, we note that a very similar result $(-8.2 \mathrm{meV})$ is obtained when the mass of protium instead of tritium is used in the calculation, which follows from the fact that the system is close to the classical limit and the classical canonical partition function is independent of the mass of the atoms.

\section{S2.1.2 Ar dimer}

Applying the treatment in Section S2.1.1 to the Ar dimer we find the values 1.9183 and 1.5700 for $\frac{q_{\mathrm{vib}, 1}}{q_{\mathrm{vib}, 0}}$ and $\frac{q_{\mathrm{rot}, 1}}{q_{\mathrm{rot}, 0}}$, respectively. Here, the reduced mass of $\mu=3.32 \cdot 10^{-26} \mathrm{~kg}$, the harmonic vibrational frequency of the relaxed dimer of $\omega=1.37 \mathrm{THz}$, and the symmetry

number $\sigma=2$ were used, while the expansion coefficients $c_{n}\left(\right.$ in $\mathrm{eV} \AA^{\mathrm{n}+1}$ ) obtained by fitting the full potential $V_{1}$ (Figure 3) by the inverse power expansion were -7.4, 192.8, -2053.2, $11478.2,-35386.0,56684.2$ and -36523.4 for $n \in[0,6]$, respectively. As in the case of HF, the 1D Schrödinger eq. (S15) was solved numerically by the Fourier grid Hamiltonian method with a regular grid of 129 points. These values yield $\Delta A_{0, \boldsymbol{x} \rightarrow 1}=\Delta A_{0, \boldsymbol{q} \rightarrow 1}=-0.0285 \mathrm{eV}$ in excellent agreement with the reference value of $-27.4 \mathrm{meV}$ reported in our article.

\section{S2.1.3 Ethane molecule}

Assuming that the individual degrees of freedom are independent, the free energy difference between the model harmonic in internal coordinates $(0, \boldsymbol{q})$ and the hindered rotor model (1) of ethane can be computed as follows:

$$
\Delta A_{0, \boldsymbol{q} \rightarrow 1}=-k_{\mathrm{B}} T \ln \frac{q_{\mathrm{hr}, 1}}{q_{\mathrm{vib}, 0, \boldsymbol{q}}^{\prime}}
$$


where $q_{\mathrm{hr}, 1}$ is the partition function of the $1 \mathrm{D}$ hindered rotor and $q_{\mathrm{vib}, 0, \boldsymbol{q}}^{\prime}$ is that for its harmonic approximation. ${ }^{6}$ The overall translations and rotations as well as all vibrational degrees of freedom apart from the specific vibrational mode corresponding to the frustrated internal rotation in the harmonic model are assumed to be identical in the models 0 and 1, hence their contributions to the partition functions cancel. The quantum mechanical expressions for the involved partition functions are ${ }^{6}$

$$
q_{\mathrm{vib}, 0, \boldsymbol{q}}^{\prime}=\frac{\exp \left(-\frac{\hbar \omega_{\boldsymbol{q}}}{2 k_{\mathrm{B}} T}\right)}{1-\exp \left(-\frac{\hbar \omega_{\boldsymbol{q}}}{k_{\mathrm{B}} T}\right)} \quad \text { and } \quad q_{\mathrm{hr}, 1}=\frac{1}{\sigma_{r}} \sum_{j} g_{j} \exp \left(-\beta \varepsilon_{j}\right)
$$

where $\sigma_{r}=3$ is the symmetry number of the internal rotation, $g_{j}$ is the degeneracy of the quantum state $j$ with eigenenergy $\varepsilon_{j}$, and $\frac{\omega_{q}}{2 \pi}=\frac{\sigma_{r}}{2 \pi} \sqrt{\frac{W}{2 I}}=5.04 \mathrm{THz}$ (see, e.g., McClurg et al. $^{8}$ ) is the harmonic frequency of hindered rotor defined via potential energy barrier for the internal rotation $(W=0.110 \mathrm{eV})$ and the effective moment of inertia $(I)$. Neglecting Coriolis contributions, the latter is computed using $^{9}$

$$
\frac{1}{I}=\frac{1}{I_{G_{1}}}+\frac{1}{I_{G_{2}}} \quad \text { with } \quad I_{G_{j}}=\sum_{i \in G_{j}} m_{i} d_{i}^{2}
$$

where $G_{1}$ and $G_{2}$ indicate the two rotating groups of atoms (here both $G_{1}$ and $G_{2}$ represent the two methyl groups of ethane), $d_{i}$ is the distance between the rotation axis (in this case passing through the $\mathrm{C}-\mathrm{C}$ bond) and the atom $i$ with mass $m_{i}$ that belongs to the group $G_{j}$. The effective moment of inertia $\left(I=7.89 \cdot 10^{-47} \mathrm{~kg} \mathrm{~m}^{2}\right)$ is then used in eq. (S16) for the internal rotation involving two methyl groups. The eigenenergies $\varepsilon_{j}$ are obtained by numerically solving the periodic 1D Schrödinger equation:

$$
-\frac{\hbar^{2}}{2 \mu} \frac{d^{2}}{d \tau^{2}} \Psi+V_{1}(\tau) \Psi=\varepsilon \Psi
$$

To this end a method described in chapter 7 of Kittel (2005) ${ }^{10}$ (section 'Solution of the central equation') was adapted whereby the full potential $V_{1}$ (Figure 4) was expressed in the 
form of a Fourier series with 9 terms obtained by fitting the potential energies evaluated on a $1 \mathrm{D}$ grid. With $T=400 \mathrm{~K}$ we obtained $\frac{q_{\mathrm{hr}, 1}}{q_{\mathrm{vib}, 0, q}}=1.119$ yielding in turn the result $\Delta A_{0, q \rightarrow 1}=-0.0039 \mathrm{eV}$ which is in excellent agreement with our reference value of $-4.1 \mathrm{meV}$. The model described in Hill $(1986)^{11}$ represents an approximation to the treatment described above. In particular, only a single dominant term of the Fourier expansion $V_{1} \approx \frac{W}{2}\left(1-\cos \left(\sigma_{r} \tau\right)\right)$. Since the actual $V_{1}$ is very well represented by a single cosine function, the result of $\Delta A_{0, q \rightarrow 1}=-0.0044 \mathrm{eV}$ obtained by the quantum mechanical treatment of a potential of the latter form is very similar to the less approximate result discussed above.

Finally, the free energy contribution due to the transformation from the potential harmonic in Cartesian coordinates to that harmonic in internal coordinates can be computed as follows

$$
\Delta A_{0, \boldsymbol{x} \rightarrow 0, \boldsymbol{q}}=-k_{\mathrm{B}} T \ln \frac{q_{\mathrm{vib}, 0, \boldsymbol{q}}}{q_{\mathrm{vib}, 0, \boldsymbol{x}}^{\prime}}
$$

where the quantum mechanical expression for $q_{\mathrm{vib}, 0, \boldsymbol{q}}$ is given by eq. (S19) and that for $q_{\mathrm{vib}, 0, \boldsymbol{x}}$ writes $^{6}$

$$
q_{\mathrm{vib}, 0, \boldsymbol{x}}^{\prime}=\frac{\exp \left(-\frac{\hbar \omega_{x}}{2 k_{\mathrm{B}} T}\right)}{1-\exp \left(-\frac{\hbar \omega_{x}}{k_{\mathrm{B}} T}\right)}
$$

with the harmonic frequency $\frac{\omega_{x}}{2 \pi}=5.12 \mathrm{THz}$ computed by diagonalization of the dynamical matrix defined in Cartesian coordinates and selection of the vibrational mode corresponding to the frustrated rotation. The value of $\frac{q_{v i b, 0, \boldsymbol{q}}}{q_{\mathrm{vib}, 0, \boldsymbol{x}}^{\prime}}=1.015$ is leading to $\Delta A_{0, \boldsymbol{x} \rightarrow 0, \boldsymbol{q}}=-0.0005 \mathrm{eV}$, which is basically identical with the classical value obtained via TI that we report in the main text. 


\section{S2.2 Classical vs. quantum harmonic approximation for adsorp- tion problems}

At very low temperatures, the free energies of matter are dominated by quantum effects which are not considered in classical theory. Deviations between quantum and classical descriptions vanish with increasing temperature as they approach the same value.

In our article we use classical DFT-based molecular dynamics (MD) and thermodynamic integration (TI) from the classical harmonic approximation to the interacting system to de-

termine classical anharmonic corrections. While the anharmonic correction from classical MD is always classical, we are free to choose between classical and quantum harmonic approximation. For conceptual clarity we decided to use the classical harmonic approximation.

In this section we show that for the adsorption free energies at the temperature considered in our adsorption studies (200 K) there is essentially no significant difference between the free energy contributions of classical and quantum harmonic approximation. Quantum harmonic vibrational contributions to the free energy were computed using the thermochemistry routines of the atomic simulation environment (ASE) ${ }^{12}$ based on equations of chapter 10 in Cramer (2004). ${ }^{13}$ The classical vibrational contribution to the free energy of a system with $N_{\text {vib }}$ degrees of freedom (number of harmonic frequencies) is computed by eq. (S24).

$$
A_{\text {vib }}=-k_{\mathrm{B}} T \sum_{i=1}^{N_{\mathrm{vib}}} \ln \frac{k_{\mathrm{B}} T}{\hbar \omega_{i}}
$$

The harmonic vibrational free energy contributions to the individual components of the adsorption free energy (eq. (26) in our article) as a function of temperature are depicted in Figure S2. The harmonic vibrational free energy contribution to the adsorption free energy as a function of temperature is shown in Figure S3. Classical and quantum theory for individual components approach each other rather slowly with increasing temperature. However, the significant difference of classical and quantum description above $100 \mathrm{~K}$ converges to negligible values $(<2 \mathrm{meV}$ at $200 \mathrm{~K})$ for the adsorption free energy itself. In consequence, the classical 

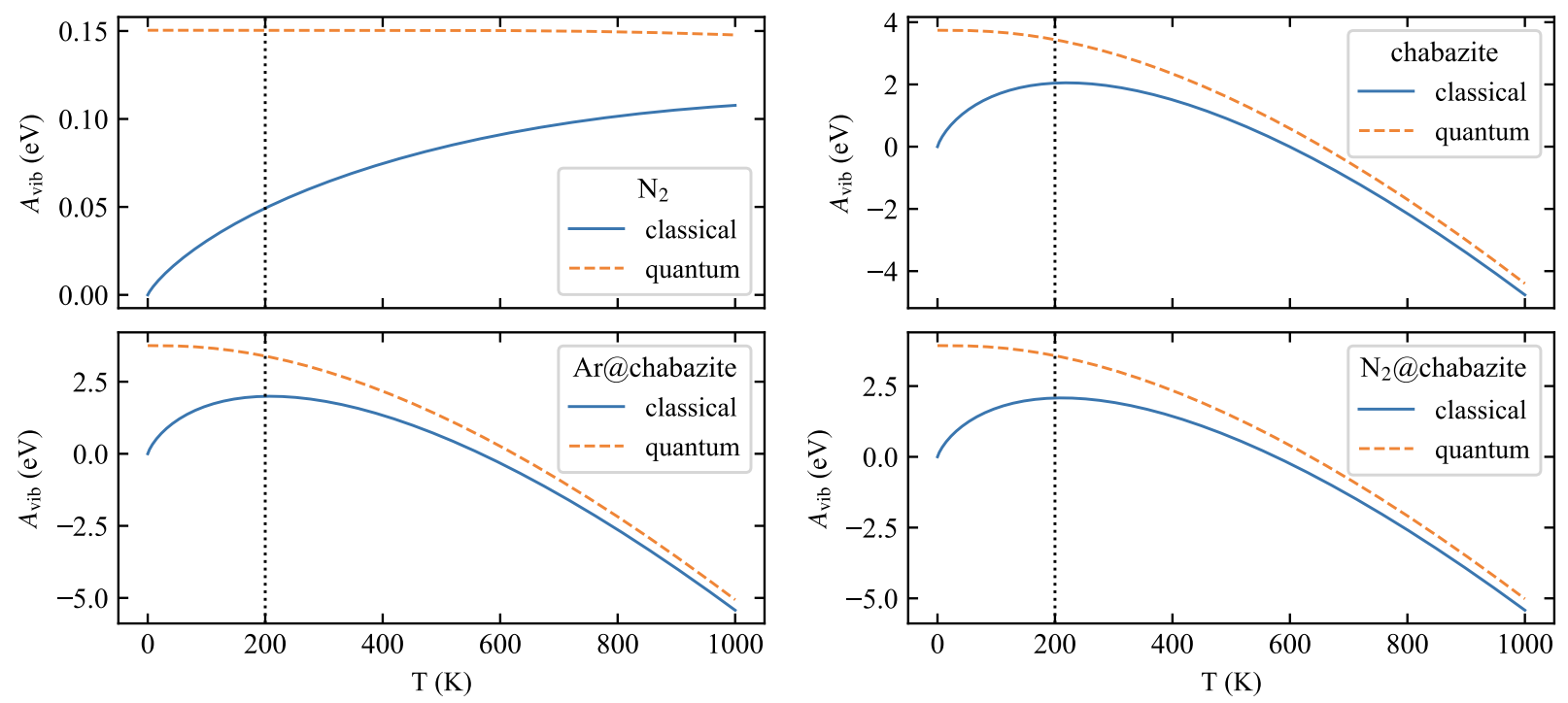

Figure S2: Harmonic vibrational free energy contribution to individual components (molecular $\mathrm{N}_{2}$, empty chabazite, adsorbed $\mathrm{Ar}$ and adsorbed $\mathrm{N}_{2}$ ) which are needed for the calculation of adsorption free energies. Consistent with our MD simulations at $200 \mathrm{~K}$, the mass of tritium was used for $\mathrm{H}$ atoms.
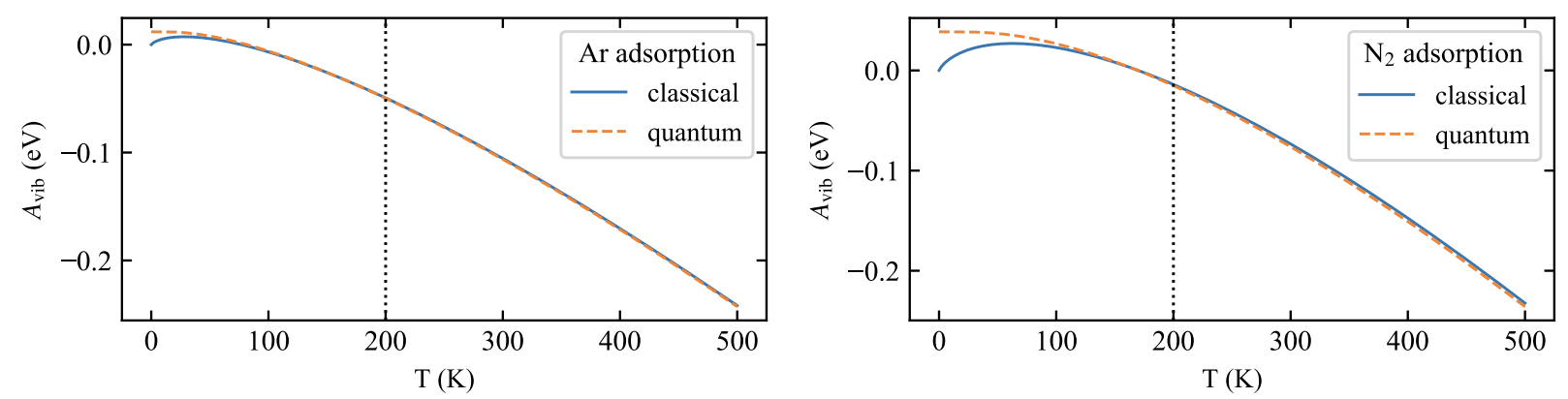

Figure S3: Harmonic free energy contribution to the adsorption free energy of Ar, i.e., $\mathrm{N}_{2}$ in chabazite zeolite. Consistent with our MD simulations at $200 \mathrm{~K}$, the mass of tritium was used for $\mathrm{H}$ atoms. 
limit is adequate for the adsorption studies discussed in our work.

\section{S3 Computational details}

\section{S3.1 Numerical integration}

Throughout our article the composite Simpson integration adapted for irregular spaced data $^{14-16}$ was used to evaluate the integrals over the $\lambda$-path involved in our TI approach. In this method, the integral $I$ is determined from

$$
I=\int_{a}^{b} f(\lambda) d \lambda \approx \sum_{i=0}^{N / 2-1}\left(\alpha_{i} f_{2 i+2}+\beta_{i} f_{2 i+1}+\eta_{i} f_{2 i}\right)
$$

where $f_{k}$ denotes the value of the integrand (in our case corresponding to $\left\langle V_{1}-V_{0, q}\right\rangle_{\lambda}$, i.e., $\left.\left\langle V_{0, \boldsymbol{q}}-V_{0, \boldsymbol{x}}\right\rangle_{\lambda}\right)$ evaluated at the $k$-th of a total of $N$ grid points defined for different values of $\lambda$. The coefficients $\alpha_{i}, \beta_{i}$ and $\eta_{i}$ are defined in eqs. (S26) to (S28).

$$
\begin{aligned}
\alpha_{i} & =\frac{2 h_{2 i+1}^{3}-h_{2 i}^{3}+3 h_{2 i} h_{2 i+1}^{2}}{6 h_{2 i+1}\left(h_{2 i+1}+h_{2 i}\right)} \\
\beta_{i} & =\frac{h_{2 i+1}^{3}+h_{2 i}^{3}+3 h_{2 i+1} h_{2 i}\left(h_{2 i+1}+h_{2 i}\right)}{6 h_{2 i+1} h_{2 i}} \\
\eta_{i} & =\frac{2 h_{2 i}^{3}-h_{2 i+1}^{3}+3 h_{2 i+1} h_{2 i}^{2}}{6 h_{2 i}\left(h_{2 i+1}+h_{2 i}\right)}
\end{aligned}
$$

Note that for even $N$ the last interval is treated with the coefficients in eqs. (S29) to (S31).

$$
\begin{aligned}
& \alpha=\frac{2 h_{N-1}^{2}+3 h_{N-1} h_{N-2}}{6\left(h_{N-2}+h_{N-1}\right)} \\
& \beta=\frac{h_{N-1}^{2}+3 h_{N-1} h_{N-2}}{6 h_{N-2}} \\
& \eta=\frac{h_{N-1}^{3}}{6 h_{N-2}\left(h_{N-2}+h_{N-1}\right)}
\end{aligned}
$$




\section{S3.2 Statistical error estimation}

The standard error $\epsilon_{I}$ in $I$ is estimated from error propagation theory applied to eq. (S25):

$$
\epsilon_{I}=\sqrt{\sum_{k=1}^{N}\left(\frac{\partial I}{\partial f_{k}} \epsilon_{f_{k}}\right)^{2}} .
$$

In our calculations, the partial derivatives $\frac{\partial I}{\partial f_{k}}$ were computed numerically via finite differences with a numerical step of $\Delta f \approx 10^{-3}$. Upon elimination of the time correlation from the MD data via the procedure of block averaging, ${ }^{17} \epsilon_{f_{k}}$ at each grid point $k$ was obtained.

\section{S3.3 Structural model for the adsorption studies}

The adsorption studies presented in Section 3.2 of our article were computed with the primitive unit cell of acid chabazite with a $\mathrm{Si} / \mathrm{Al}$ ratio of 11 (see Figure $\mathrm{S} 4$ ). The acid proton was

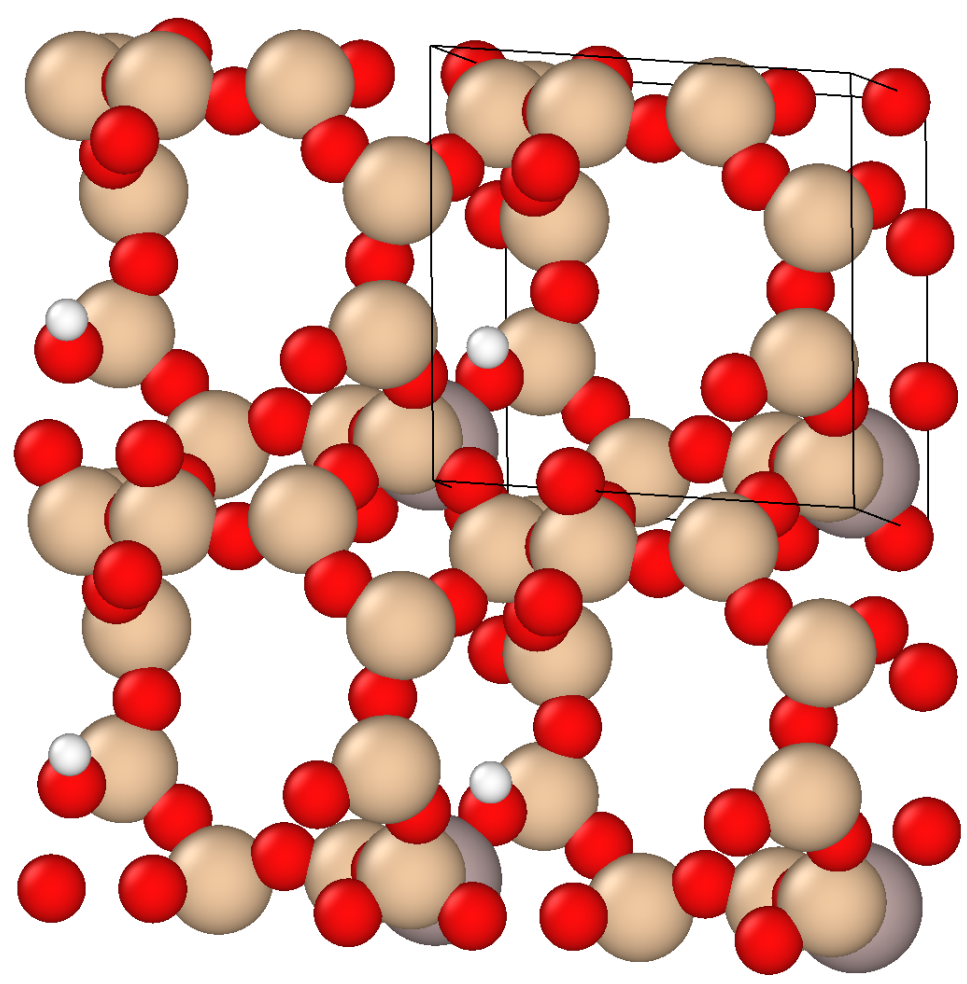

Figure S4: The frame in the upper right corner marks the boundaries of the primitive unit cell of chabazite. Color code: H (white), O (red), Si (beige), Al (gray). 
located in position $\mathrm{O}(1)$ according to the nomenclature of Jeanvoine et al., ${ }^{18}$ which belongs to one of the most populated proton sittings on chabazite. ${ }^{19}$ The lattice parameters have been derived from the experimental geometry determined for a highly siliceous form (H-SSZ-13) of chabazite ${ }^{20}$ (space group $R \overline{3} m$ ) with lattice constants of $a=9.338 \AA$ and $\alpha=95.0^{\circ}$. We used the same unit cell also in related calculations of electronic, vibrational and rotational contributions for free energies of the isolated adsorbates particles Ar and $\mathrm{N}_{2}$.

\section{S4 Regression of experimental adsorption free energies}

Adsorption free energies of $\mathrm{Ar}$ and $\mathrm{N}_{2}$ in acid chabazite were measured by Barrer and Davies $^{21}$ for a range of temperatures $\left(138 \mathrm{~K}\right.$ to $195 \mathrm{~K}$ for $\mathrm{Ar}$ and $78 \mathrm{~K}$ to $210 \mathrm{~K}$ for $\mathrm{N}_{2}$ ). Their experimental data plotted in Figure S5 exhibit a pronounced linear trend, which was used to determine $\Delta_{\text {ads }} G$ at $200 \mathrm{~K}$.

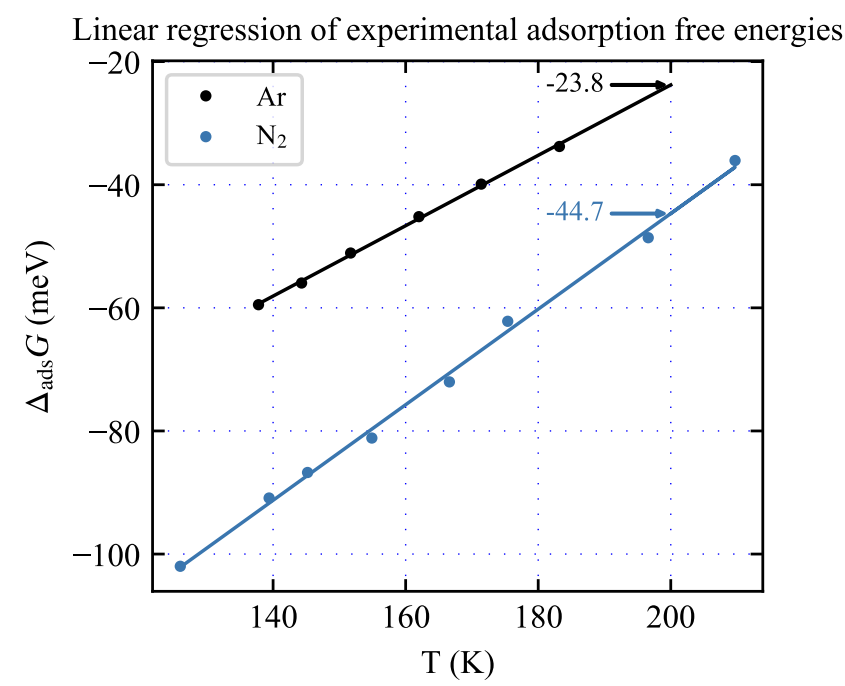

Figure S5: Linear regression of experimental adsorption free energies measured by Barrer and Davies ${ }^{21}$ for $\mathrm{Ar}$ and $\mathrm{N}_{2}$ in acid chabazite. Adsorption free energies at $200 \mathrm{~K}$ are annotated. 


\section{S5 Practical information}

\section{S5.1 How to perform a TI MD in VASP - a brief workflow}

The TI method discussed in our article is available in VASP version 6.x, the patch introducing the method to version 5.4 .4 can be provided by the authors upon request. The current workflow is presented in Scheme S1. The energy of the input geometry is opti-
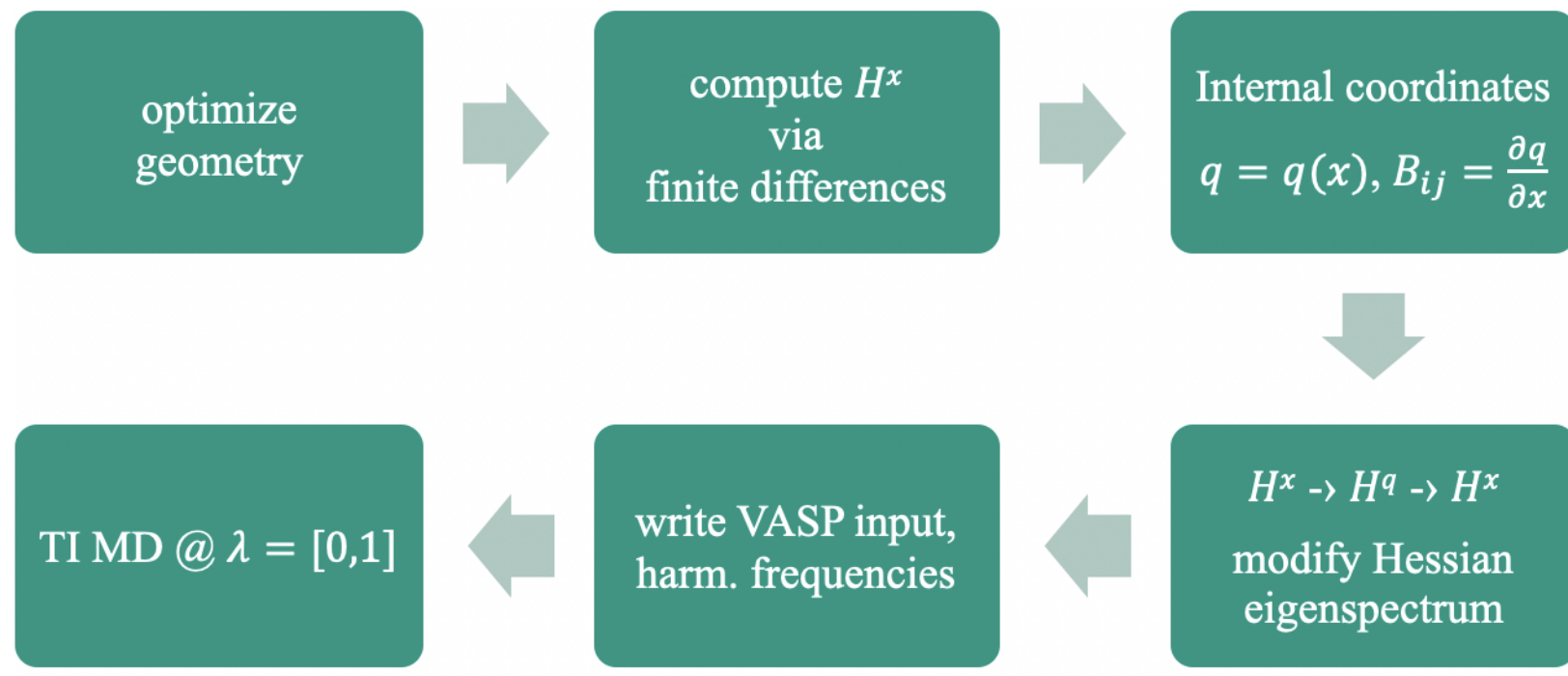

\section{$H^{x} \rightarrow H^{q} \rightarrow H^{x}$}

modify Hessian

eigenspectrum

harmonic force field

to force field

TI MD@ $\lambda \in[0,1]$

Scheme S1: Current workflow to compute anharmonic corrections via TI MD in VASP.

mized with a tight relaxation criterion (here all forces acting on the atoms were required to be below $0.005 \mathrm{eV} \AA^{-1}$ ) and the numerical Hessian matrix $\underline{\boldsymbol{H}}^{\boldsymbol{x}}$ is computed using a finite differences scheme. Next, a set of redundant rotationally and translationally invariant internal coordinates $\boldsymbol{q}$ is defined and used to project out the overall rotations and translations via forward (Cartesian to internal) and backward (internal to Cartesian) coordinates transformation. If needed, the eigenvalues of the Hessian matrix are modified 
as described in Section 2.1.2 of the main text, and the free energy contribution $A_{0, \boldsymbol{x}}$ is computed. In order to perform the TI calculations with VASP, the files defining the internal coordinates and the Hessian matrix must be provided. The former is specified in the file ICONST (https://www.vasp.at/wiki/index.php/ICONST), whereby the status of coordinates active in TI must be set to 3 , while the latter is defined in the file HESSEMAT generated from the OUTCAR from the vibrational analysis. Finally, the value of $\lambda$ is specified (along with the standard MD keywords) via the tag TILAMBDA. The potential energy differences $V_{1}-V_{0, \boldsymbol{q}}$ are written for every MD step in the file REPORT (https://www.vasp.at/wiki/index.php/REPORT).

Python tools to generate the files ICONST and HESSEMAT, as well as the tools to perform the force field to force field transformation $A_{0, \boldsymbol{x} \rightarrow 0, \boldsymbol{q}}$ numerically are available upon request.

\section{References}

(1) Fogarasi, G.; Zhou, X.; Taylor, P. W.; Pulay, P. The Calculation of Ab Initio Molecular Geometries: Efficient Optimization by Natural Internal Coordinates and Empirical Correction by Offset Forces. J. Am. Chem. Soc. 1992, 114, 8191-8201.

(2) Baker, J. Techniques for Geometry Optimization: A Comparison of Cartesian and Natural Internal Coordinates. J. Comput. Chem. 1993, 14, 1085-1100.

(3) Bakken, V.; Helgaker, T. The Efficient Optimization of Molecular Geometries Using Redundant Internal Coordinates. J. Chem. Phys. 2002, 117, 9160-9174.

(4) Bučko, T.; Hafner, J.; Ángyán, J. G. Geometry Optimization of Periodic Systems Using Internal Coordinates. J. Chem. Phys. 2005, 122, 124508.

(5) Bučko, T. Transition State Optimization of Periodic Systems Using Delocalized Internal Coordinates. Theor. Chem. Acc. 2018, 137, 164. 
(6) Jensen, F. Introduction to Computational Chemistry, 2nd ed.; John Wiley \& Sons: Chichester, England ; Hoboken, NJ, 2007.

(7) Marston, C. C.; Balint-Kurti, G. G. The Fourier Grid Hamiltonian Method for Bound State Eigenvalues and Eigenfunctions. J. Chem. Phys. 1989, 91, 3571-3576.

(8) McClurg, R. B.; Flagan, R. C.; Goddard III, W. A. The Hindered Rotor Density-ofStates Interpolation Function. J. Chem. Phys. 1997, 106, 6675-6680.

(9) Pfaendtner, J.; Yu, X.; Broadbelt, L. J. The 1-D Hindered Rotor Approximation. Theor. Chem. Acc. 2007, 118, 881-898.

(10) Kittel, C. Introduction to Solid State Physics, 8th ed.; Wiley: Hoboken, NJ, 2005.

(11) Hill, T. L. An Introduction to Statistical Thermodynamics; Dover Publications: New York, 1986.

(12) Hjorth Larsen, A.; Jørgen Mortensen, J.; Blomqvist, J.; Castelli, I. E.; Christensen, R.; Dułak, M.; Friis, J.; Groves, M. N.; Hammer, B.; Hargus, C.; Hermes, E. D.; Jennings, P. C.; Bjerre Jensen, P.; Kermode, J.; Kitchin, J. R.; Leonhard Kolsbjerg, E.; Kubal, J.; Kaasbjerg, K.; Lysgaard, S.; Bergmann Maronsson, J.; Maxson, T.; Olsen, T.; Pastewka, L.; Peterson, A.; Rostgaard, C.; Schiøtz, J.; Schütt, O.; Strange, M.; Thygesen, K. S.; Vegge, T.; Vilhelmsen, L.; Walter, M.; Zeng, Z.; Jacobsen, K. W. The Atomic Simulation Environment-a Python Library for Working with Atoms. J. Phys. Condens. Mat. 2017, 29, 273002.

(13) Cramer, C. J. Essentials of Computational Chemistry: Theories and Models, 2nd ed.; Wiley: Chichester, West Sussex, England ; Hoboken, NJ, 2004.

(14) Shklov, N. Simpson's Rule for Unequally Spaced Ordinates. Am. Math. Mon. 1960, 67, 1022. 
(15) Press, W. H., Ed. Numerical Recipes: The Art of Scientific Computing, 3rd ed.; Cambridge University Press: Cambridge, UK; New York, 2007.

(16) Cameron, R., B. Numerically Integrating Irregularly-Spaced (x, y) Data. Math. Enthus. 2014,11 .

(17) Flyvbjerg, H.; Petersen, H. G. Error Estimates on Averages of Correlated Data. J. Chem. Phys. 1989, 91, 461-466.

(18) Jeanvoine, Y.; Ángyán, J. G.; Kresse, G.; Hafner, J. Brønsted Acid Sites in HSAPO-34 and Chabazite: An Ab Initio Structural Study. J. Phys. Chem. B 1998, 102, 55735580.

(19) Smith, L.; Davidson, A.; Cheetham, A. A Neutron Diffraction and Infrared Spectroscopy Study of the Acid Form of the Aluminosilicate Zeolite, Chabazite (H-SSZ-13). Catal. Lett. 1997, 49, 143-146.

(20) Zones, S.; Van Nordstrand, R. Novel Zeolite Transformations: The Template-Mediated Conversion of Cubic P Zeolite to SSZ-13. Zeolites 1988, 8, 166-174.

(21) Barrer, R. M.; Davies, J. A. Sorption in Decationated Zeolites. I. Gases in HydrogenChabazite. Proc. Roy. Soc. Lond. A. 1970, 320, 289-308. 